\title{
A novel MAs(III)-selective ArsR transcriptional repressor
}

\author{
J. Chen, V.S. Nadar \& B.P. Rosen \\ Department of Cellular Biology and Pharmacology, Herbert Wertheim College of Medicine, \\ Florida International University, Miami, Florida, USA
}

\begin{abstract}
All heavy metals can lead to toxicity and oxidative stress when taken up in excessive amounts, imposing a serious threat to the environment and human health. Expression of genes for resistance to heavy metals and metalloids is frequently transcriptionally regulated by the toxic ions themselves. Arsenic is a ubiquitous, naturally occurring toxic metalloid widely distributed in soil and groundwater. Microbes biotransform both pentavalent $(\mathrm{As}(\mathrm{V}))$ and trivalet $(\mathrm{As}(\mathrm{III}))$ arsenic into more the toxic methylated metabolites methylarsenite acid (MAs(III)) and dimethylarsenite (DMAs(III)). Environmental arsenic is sensed by members of the ArsR/SmtB family of metalloregulatory proteins. The arsR gene is autoregulated and is typically part of an operon that contains other ars genes involved in arsenic detoxification. To date, every identified ArsR is regulated by inorganic As(III). Here we described a novel ArsR from Shewanella putrefaciens that is specific for MAs(III) and does not respond to As(III). SpArsR has three conversed cysteine: Cys83, Cys101 and Cys102. Substitutions of Cys 83 with serine has no effect for ArsR activity. However, mutation of C101S and C102S mutants lost MAs(III) binding affinity, which indicates that these two cysteines are required for MAs(III) binding. SpArsR can be converted into an As(III)-responsive repressor by introduction of an additional cysteine that allows for 3-coordinate As(III) binding. Our results indicate that SpArsR evolved selectivity for MAs(III) over As(III) in order to control expression of genes for MAs(III) detoxification.
\end{abstract}

\section{INTRODUCTION}

Nearly every organism has genetic mechanisms for arsenic resistance. Their genes are usually organized in ars operons, which are found either on the chromosome or on plasmids (Zhu et al., 2014). Typically ars operons are regulated by an ArsR As(III)-responsive transcriptional repressor. Recently a parallel biocycle for organoarsenicals has been identified (Chen $e t$ $a l ., 2014)$. In response to this environmental pressure, other bacteria have evolved mechanisms to detoxify MAs(III). These organoarsenical detoxification genes are in ars operons regulated by a homodimeric As(III)-responsive ArsR repressor. Three different ArsRs have been identified that have three types of As(III) binding sites (Ordóñez et al., 2008). The ArsR repressor encoded by Escherichia coli plasmid R773 (termed a Type 1 site). Two other ArsR orthologs with different As(III) binding sites were identified in the ars operon of Acidithiobacillus ferrooxidans and Corynebacterium glutamicum, respectively. Each ArsR has a high-affinity As(III) binding site composed of three cysteine residues at spatially distinct locations in their three-dimensional structures (Qin et al., 2007). In this study we identified a novel MAs(III)responsive ArsR that regulates expression of the ars $P$ and arsH MAs(III) resistance genes in Shewanella putrefaciens 200 . SpArsR is induced most effectively by MAs(III), with little response to As(III). SpArsR appears to have two conversed Cys101 and Cys102, which were involved in MAs(III) induction. We postulate that SpArsR evolved selectivity for MAs(III) in order to respond to environmental MAs(III) produced by other soil microbes.

\section{METHODS/EXPERIMENTAL}

\subsection{Biosensor construction}

To analysis the ArsR regulation by arsenicals, a biosensor with $g f p$ reporter was constructed. For expression of $S p A r s R$ from $S$. putrefaciens, plasmid pBAD$S p A r s R$ was constructed. The $g f p$ reporter is under control of the Spars $P$ promoter and generated plasmid pACYC184-Pars $P$-gfp. Transcriptional activity of the biosensor was estimated from arsenical-responsive expression of $g f p$. Expression of $g f p$ was assayed from the fluorescence of cells.

\subsection{Mutagenesis of the SpArsR gene}

SparsR mutations were generated by site-directed mutagenesis using a Quick Change mutagenesis kit. The codons for residues Cys83, Cys101 and Cys102 were changed to serine codons, generating three different single cysteine SpArsR mutants.

\section{RESULTS AND DISCUSSION}

\subsection{SpArsR has a MAs(III) binding site}

In ArsRs, cysteine thiolates usually form threecoordinate complexes with As(III). In AfArsR, Cys95, Cys96 and Cys102 form the three-coordinate As(III) binding site. In the homology model of SpArsR, 


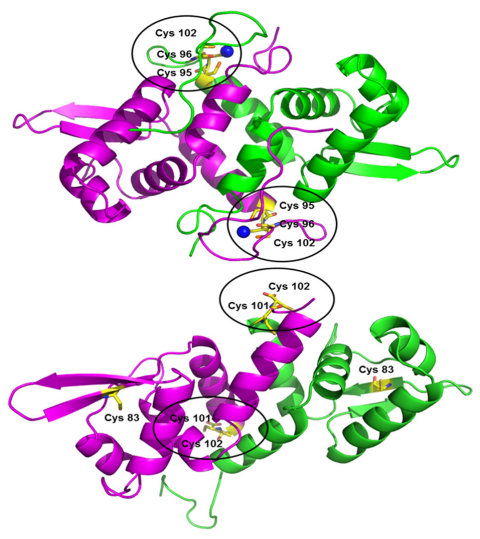

Figure 1. Homology modeling of As(III)-bound AfArsR and MAs(III)-bound SpArsR.
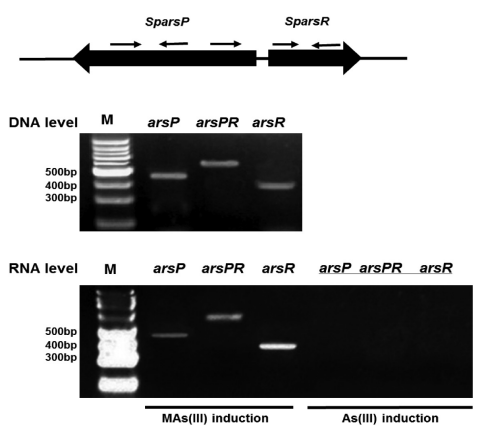

Figure 2. SparsR and $\operatorname{Spars} P$ are linked transcriptionally by MAs(III) induction.

Cys101 and Cys102 are superimposible with AfArsR Cys95 and Cys96. Cys83, which is found in putative orthologs but not in AfArsR, is located distant from Cys101 and Cys102 and would not be expected to contribute to inducer binding (Fig. 1).

\subsection{SpArsR is a MAs(III)-selective repressor}

Qualitative reverse transcription-PCR (RT-PCR) was used to assess $\operatorname{Spars} R$ and $\operatorname{Spars} P$ expression following exposure to As(III) or MAs(III). There was no detectable expression of either SparsR or SparsP expression following exposure to As(III) (Fig. 2). In contrast, both were expressed at high levels following exposure to MAs(III). Results showed that SparsR is induced by MAs(III) and not As(III) and regulates a gene for MAs(III) detoxification.

\subsection{The MAs(III) binding site in SpArsR}

Elevated $g f p$ expression indicates that $\mathrm{SpArsR}$ is highly selective for MAs(III) binding and derepresses $g f p$ expression. Cys83 is not required for MAs(III) response. Both Cys101 and Cys102 are involved in MAs(III) binding. Cells with the wild type SparsR biosensor responded only to MAs(III), and cellular $g f p$ derepression was proportional to the concentration of
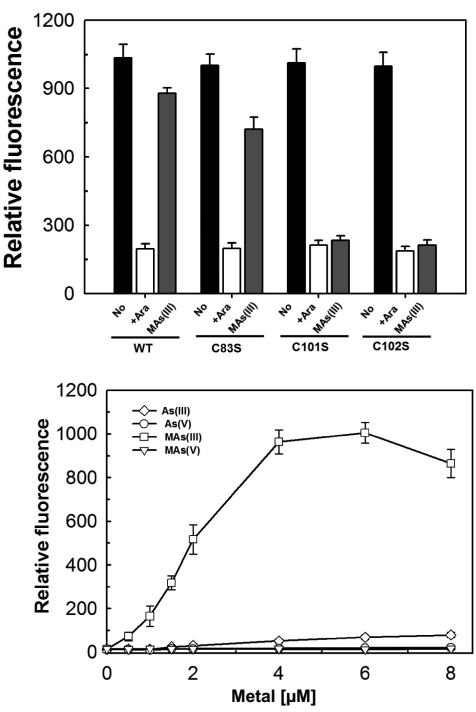

Figure 3. Binding of MAs(III) to SpArsR involves specific cysteine residues.

MAs(III). Cells with the wild type SpArsR biosensor were unresponsive to $\mathrm{MAs}(\mathrm{V})$ or $\mathrm{As}(\mathrm{V})$ and exhibited only a very low response to As(III) (Fig. 3).

\section{CONCLUSION}

The MAs(III) binding site of SpArsR appears to have evolved from an ancestor common to the AfArsR As(III) binding site. The SpArsR MAs(III) binding site appears to be the result of a loss-of-function mutation where the third As(III) ligand is no longer present. In reality, though, it is a gain-of-function mutation to provide specificity for MAs(III) that allows for transcriptional regulation of MAs(III) detoxification mechanisms.

\section{ACKNOWLEDGEMENTS}

This work was supported by National Institute of General Medical Sciences and NIH grant R01 GM55425.

\section{REFERENCES}

Chen, J., Sun, S., Li, C.Z., Zhu, Y.G. \& Rosen, B.P. 2014. Biosensor for organoarsenical herbicides and growth promoters. Environ. Sci. Technol. 48: 1141-1147.

Ordóñez, E., Thiyagarajan, S., Cook, J.D., Stemmler, T.L., Gil, J.A., Mateos, L.M. \& Rosen, B.P. 2008. Evolution of metal(loid) binding sites in transcriptional regulators. $J$. Biol. Chem. 283: 25706-25714.

Qin, J., Fu, H.L., Ye, J., Bencze, K.Z., Stemmler, T.L., Rawlings, D.E. \& Rosen, B.P. 2007. Convergent evolution of a new arsenic binding site in the ArsR/SmtB family of metalloregulators. J. Biol. Chem. 282: 34346-34355.

Zhu, Y.G., Yoshinaga, M., Zhao F.J. \& Rosen, B.P. 2014. Earth abides arsenic biotransformations. Annu. Rev. Earth Planet. Sci. 42: 443-467. 